\title{
Design of Single Input Multiple Output Buck Converter with Light Load Efficiency
}

\author{
Pragnya R Mogha, Manish Rathi
}

\begin{abstract}
The major demand for portable electronics devices such as pagers, laptops, cellular phones etc. Single input multiple output (SIMO) converter will be proposed to give different supply voltages while keep up prolong battery life. A single-discharge (SDC) control scheme is introduce to simplify the Single inductor multiple output design, attain to low cross regulation, and to support board range of loads with decent efficiency of power. A Single discharge control single input multiple output buck converter with 4 outputs composed of comparators, phased-locked loop, finite state machine (FSM) controller, and an output stage. In addition to the basic switching functions, the FSM controller provides a state skipping feature to allow no-load regulation. Single input multiple output converter is available for less cost and used for maintaining long battery life and they are growing in vast range with rich feature portable electronic device. The design of converter is designed for limited range for prolonged battery life. The SIMO converters are used for the reduction of cost and switching losses, hence improve system efficiency. In our study, we proposed buck converter topology with single input of $24 \mathrm{v}$ and multiple output of $12 \mathrm{v}$ and $5 \mathrm{v}$. The $12 \mathrm{v}$ voltage is used for electric vehicles. In electric vehicles it is used in horns and headlights. $5 \mathrm{v}$ voltage is used for small signal and stability analysis. The simulated conversion efficiency for peak power is $86 \%$ the power ranging for output is 50 to $300 \mathrm{~mW}$. The ICs exhibit has a low measured peak efficiency of 73\%and cross regulation of 0.24 $\mathrm{mV} / \mathrm{mA}$ because of parasitic bond wire resistance.The proposed system is simulated in MATLAB/Simulink software.

Keywords:DC-DC Converter, Finite State Control Controller, SIMO Converter, Portable Electronic Device, Electric Vehicle.
\end{abstract}

\section{INTRODUCTION}

Multiple independent sources voltage with dense regulation is a usual but different problem to solve in portable electronics. On another switched mode power supplies (SMPS) can be given at more efficiency but desire a valuable discrete inductor and a capacitor for single output. The other option is low drop out (LOD) regulated can be completely integrated on silicon die, but there is problem in efficiency. Therefore, for system designers the SMPS is used for heavy loads which give high efficiency, and LOD used for the lighter loads. Single inductor multiple outputs are same as SMPS designs. SIMO provide more efficiency than the SMPS devices. SIMO converter has high efficiency with low cost.

Manuscript received on June 08, 2021

Revised Manuscript received on June 12, 2021.

Manuscript published on June 30, 2021.

* Correspondence Author

Pragnya R Mogha*, PG Scholar, Department of Electrical and Electronics Engineering, PDA College of Engineering, Kalaburagi(Karnataka), India. E-mail:moghapragnya1931@gmail.com

Manish Rathi, Associate Professor, Department of Electrical and Electronics Engineering, PDA College of Engineering, Kalaburagi(Karnataka), India. E-mail:manish.rathi277@gmail.com

(C) The Authors. Published by Blue Eyes Intelligence Engineering and Sciences Publication (BEIESP). This is an open access article under the CC BY-NC-ND license (http://creativecommons.org/licenses/by-nc-nd/4.0/)
In the simple form, as shown in figure 1 SIMO converter has time multiplexed version of the pulse width modulation controlled SMPS here one inductor is shared across many outputs. There are few drawbacks. These have complex design, limited range load and cross regulation. Cross regulation is an effect where output voltage will experience voltage transient as it has multiple output if there is any change in any output there it experiences a cross regulation effect. In figure 2 the first output of SIMO converter has the control architectures. These are PWM-based control, hybrid control, and fully hysteretic control.

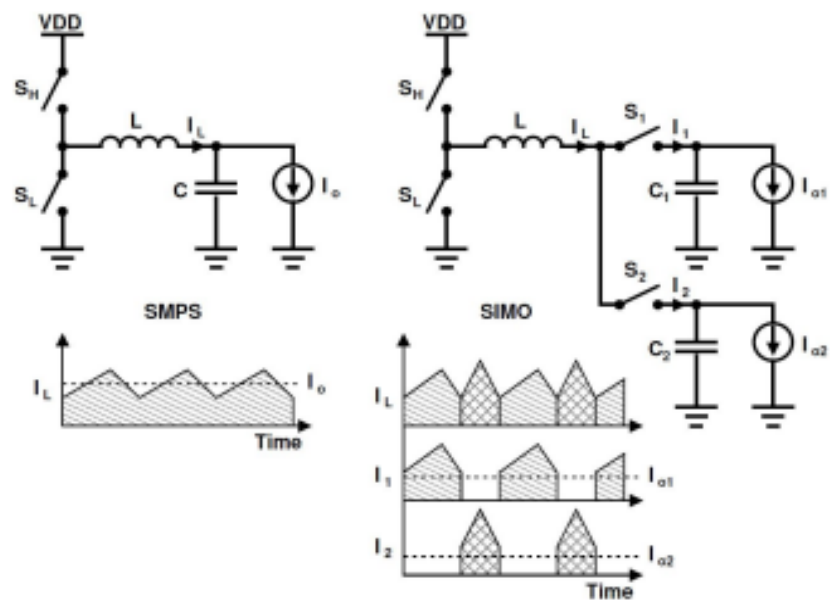

Fig 1: left side a single output SMPS buck converter is there compared with right side which has 2-output SIMO buck converter.

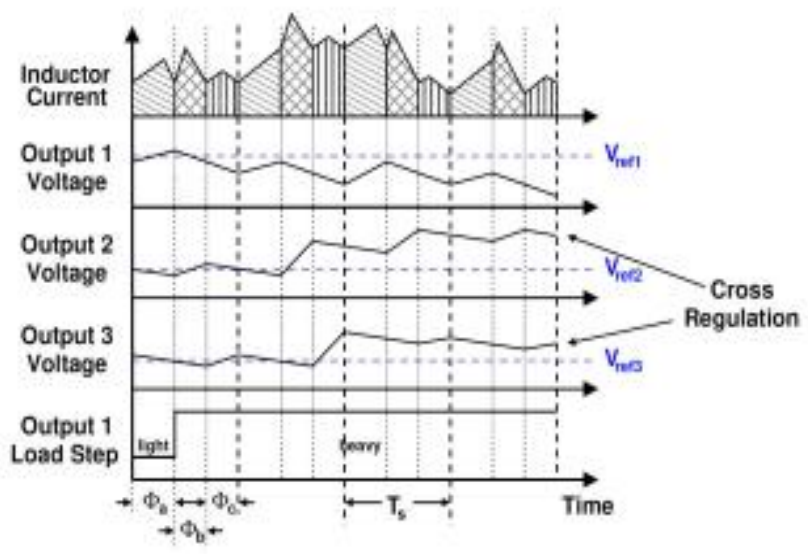

Fig 2: In a SIMO converter there is load change without cross regulation suppression.

Published By:

Blue Eyes Intelligence Engineering and Sciences Publication

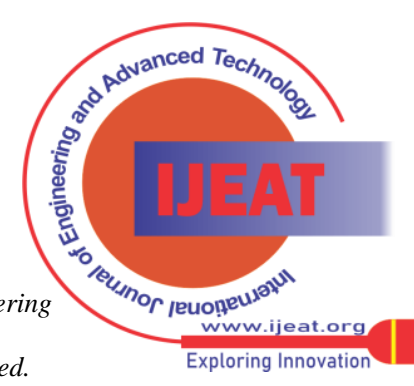




\section{PROPOSED SIMO BUCK CONVERTER TOPOLOGY}

A single discharge control (SDC) in this SIMO converter is preferred for low regulation for wide value of loads. The SDC SIMO converter is shown in the figure below with four outputs is a forward implementation to the fully hysteretic converter. There are two main phases, where in the first phase output is charged individually unless their voltage reaches to the required preferred voltage. Unlike the other works, where all outputs are charged when the rising inductor current waveform is illustrated in Fig 4. By guiding the current from high side (HS) through inductor L1 to the switches at the output S1 to S4. When the outputs are charged, the converter enters to the second phase, there is a recycling switch (RC)

and the low side switches (LS) are turn on to direct current in inductor back into the input battery. Since SIMO converters are mostly used in the mobile's applications, where the reverse current would recharge the battery or the input decoupling capacitor, at last it is used for recycling the energy. The recycling phase duration is determined by the phase looped lock which tries to align the overall switching signals (HS) with $1 \mathrm{MHz}$ reference clock in the phase. Freewheeling switch (FW) and recycling switch (RC) are added to the full hysteretic SIMO. Zero current detection is added to RC for DCM capability. The inductor current increases again to charge outputs as soon as the cycle resets. HS is the buck switch used to charge the inductor and also supply to load. FW is used to provide bypass in case the inductor is completely discharged before next switching cycle starts that is during the circuit operates in discontinuous mode. Gate driver circuit is used to drive the gate terminal of the switches when the pulses from controller is high that is 1 or on. TLP250 is used as a gate driver IC. Dynamic bulk bias (DBB) is receiving the gate signal from controller. The output capacitor is used to reduce the voltage ripples. Comparator provides the output as high when the measured voltage is higher than the reference voltage and the comparator output is low when the reference voltage is higher than measured voltage.

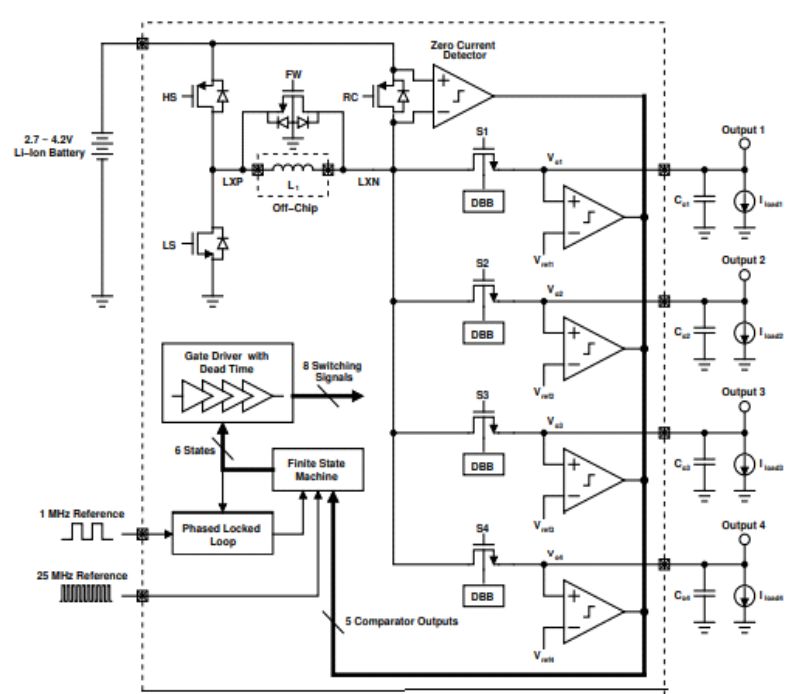

Fig 3: overview of the proposed buck converter with 4 output SIMO converters
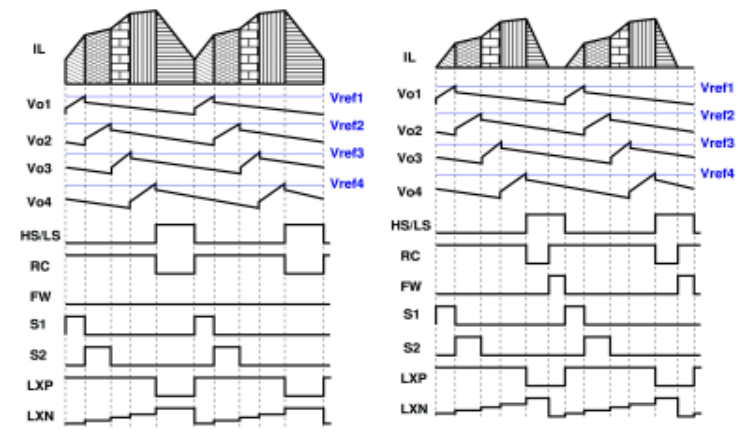

Fig 4: The proposed SDC SIMO converter waveforms it is a steady state waveform from lower to higher output voltages, inductor current, freewheeling switch, output switch1, output switch2, output switch3 and recycling switch.

For the proposed SDC SIMO converter the main component that differs from the other design is the controller. There is artificial neural network (ANN) or its simply called neural network is the computing system design to imitate the human brain process and analyzes the information. It is connected with artificial neurons and the foundation of the ANN is the artificial intelligence (AI) to resolve the problems. ANN is used to predict the output values of the given input parameters from the training values. ANN has the numerical strength that can perform more than one job at the same time; it also has the parallel processing capability.

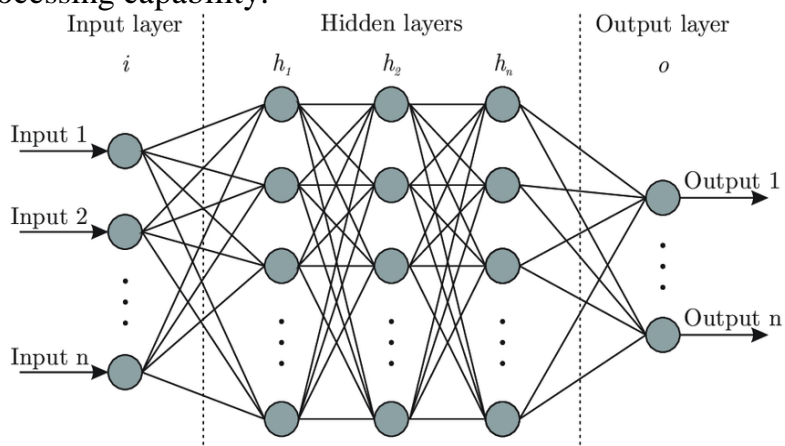

Fig 5: Architecture Artificial neural network

Figure 5 is the general architecture of ANN contains an input layer, a number of the hidden layer and the output layer. By the adaptive weights the artificial neurons are interconnected in each hidden and output layer. The training process with the input and output data are done with the weights. Every artificial neuron has an activation function which has a range of $(-1,1)$. The definition of ANN includes the number of inputs, outputs and hidden neurons the number of hidden layers ${ }^{[8]}$ The universal approximation theorem ${ }^{[9]}$ statesthat a feed-forward network with a single hidden layer containing a finite number of neurons can approximate continuous functions on compact subsets of $\mathrm{R}$ $n$, being $n$ the number of inputs. ANN design is based on the error and trail by the complexity of each case it has two methods first to determine the number of hidden layers coarse calibration is made a second one should train the increasing number of hidden neurons until these achieve these desired performances.

Published By:

Blue Eyes Intelligence Engineering and Sciences Publication

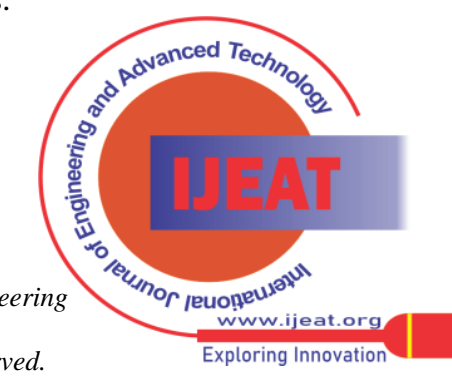




\section{EXPERIMENTAL AND SIMULATION RESULTS}

Table I: Simulation and Experimentation Parameters ${ }^{[1]}$

\begin{tabular}{|l|l|}
\hline & work \\
\hline Process & $0.18 \mu \mathrm{m}$ \\
\hline Control method & SDC \\
\hline Topology & 4 buck outputs \\
\hline Supply voltage & $24 \mathrm{v}$ \\
\hline Frequency (Mhz) & 1 \\
\hline Inductor & $4.7 \mu \mathrm{H}$ \\
\hline Capacitor & $10 \mu \mathrm{F}$ \\
\hline Output voltage ripple (mVpp) & $<20$ \\
\hline Load transient (mV/mA) & 0.1 \\
\hline Cross regulation (mV/mA) & 0.04 \\
\hline Max Efficiency & $86 \%$ \\
\hline DCM operation & supported \\
\hline No load operation & supported \\
\hline
\end{tabular}

The Simulink model of SIMO converter is shown in figure below there are five different loads and each load consists of $12 \mathrm{v}$ and 10 watts so that total power will be 50 watts and voltage will be $12 \mathrm{v}$. The power with each load is 10 watts so we are designing 1 buckconverter from $24 \mathrm{v}$ to $12 \mathrm{v}$ initially when switch IGBT is turned on at the time inductor will get charged and when the IGBT is turned on it can provide path for inductor current while it is discharging. In this IGBT when loads are disconnected at that time this IGBT will turn on. If any inductor current is present in the inductor that will discharge through the IGBT and provide the set back to the battery. Here for each load, we are using one switch and when voltage is above $12.1 \mathrm{v}$ the switch will be turned off and if the voltage goes below $12 \mathrm{v}$ the switch will get turned on. For all the loads we are using same setup. The controller here we are using is one state flow method, in this voltage measuring is done which provides as an input. Here we are providing two conditions one is voltage above $12.1 \mathrm{v}$ that is $\mathrm{v}=0$ the switch is off that is $\mathrm{y}=0$ and the second condition is when voltage goes below $12.1 \mathrm{v}$ then $\mathrm{y}=1$ the switch is on. Between the 12 to $12.1 \mathrm{v}$ the previous state will occur. If previous state is on then it continuous to be on if the previous state is off then it continuous to be off, for all the loads we are using same setup. Here we are measuring, checking and combining all this system to form one subsystem here we are using one logic operator that is AND operator so all the switches are turned off in that time the IGBT1 will be turned off in order to discharge the inductor and charge the battery. Now we can run the simulation

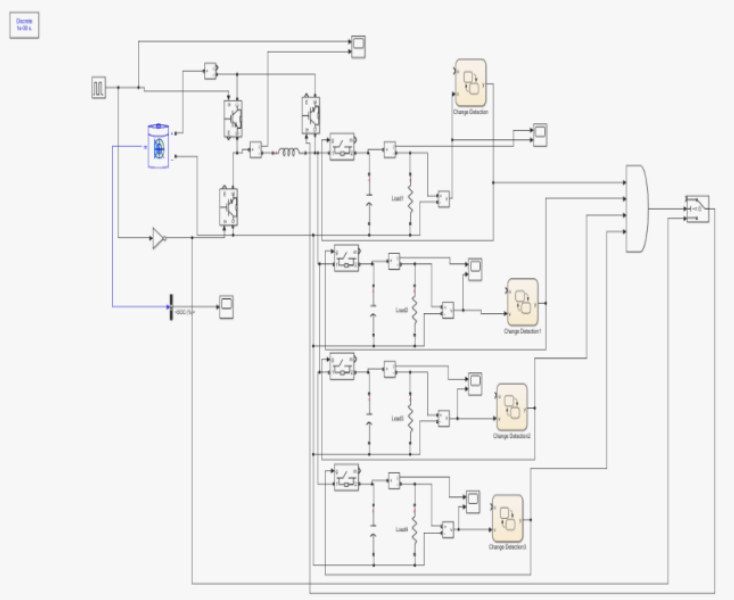

Fig 6: simulation model for the proposed topology:

Retrieval Number: 100.1/ijeat.E27870610521

Published By:

Blue Eyes Intelligence Engineering and Sciences Publication
Above Figure is load voltages in current. first waveform is of current second is of voltage there is oscillation of voltage of $12.1 \mathrm{v}$. when oscillation reaches $12.1 \mathrm{v}$ the switch is turned off and voltage will be reduced and again it will increase when it is turned on. There will be oscillation between 12 to $12.1 \mathrm{v}$

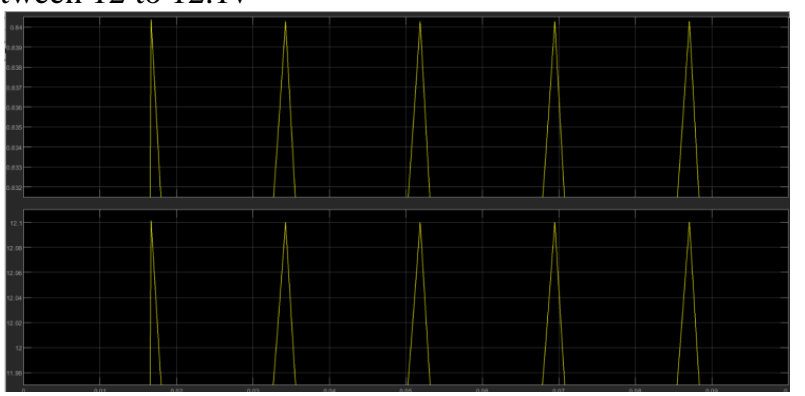

Same voltage and current time taken is nearly is $0.1 \mathrm{~s}$ to reach the peak value. for reducing the time and oscillation ANN control is used

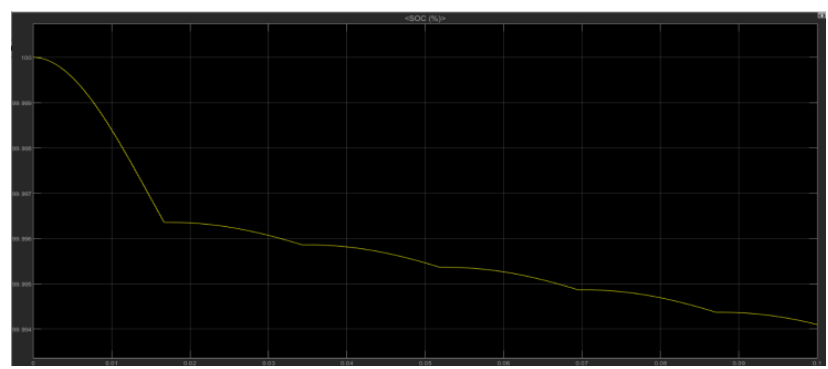

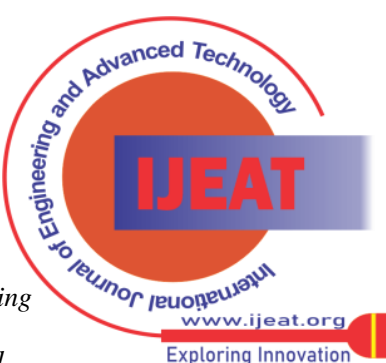


We have tried for different loads; we are using four loads instead of 10 watts some 80 watts that is each of 15 watts. checking and measuring there is curvy wave. Compared to normal conditions again it reduces the voltage. Here the called range will be 12.1 To $11.5 \mathrm{v}$. this all we have to avoid using ANN control to reduce the disturbance. if we change one load the efficiency of all other load changes. Controller is very simple; we checked the two conditions it. It is very simple and very quick here the voltage is reduced when switch is turned on. if the voltage is high, we should turn on the switch.

\section{A. Inductor Selection:}

$$
\mathrm{L}=\frac{\mathrm{V}_{\text {OUT }} \times\left(\mathrm{V}_{\mathrm{IN}}-\mathrm{V}_{\text {OUT }}\right)}{\Delta \mathrm{I}_{\perp} \times f_{\mathrm{S}} \times \mathrm{V}_{\mathrm{JN}}}
$$

VIN = typical input voltage VOUT = desired output voltage $\mathrm{fS}=$ minimum switching frequency of the converter $\Delta \mathrm{IL}=$ estimated inductor ripple current.

\section{B. Capacitor selection: [11]}

The minimum value for the input capacitor is normally given in the data sheet. This minimum value is necessary to stabilize the input voltage due to the peak current requirement of a switching power supply. The best practice is to use low-equivalent series resistance (ESR) ceramic capacitors. The dielectric material must be X5R or better. Otherwise, the capacitor loses much of its capacitance due to dc bias or temperature. The value can be increased if the input voltage is noisy.

$$
\mathrm{C}_{\text {OUT(min) }}=\frac{\Delta \mathrm{L}}{8 \times f_{\mathrm{S}} \times \Delta \mathrm{V}_{\text {OUT }}}
$$

COUT $(\min )=$ minimum output capacitance

$\Delta \mathrm{IL}=$ estimated inductor ripple current

$\mathrm{fS}=$ minimum switching frequency of the converter $\Delta$ VOUT $=$ desired output voltage ripple

\section{CONCLUSION}

The experimental result is to design single input multiple output buck converter with light load efficiency. The supply voltage is $24 \mathrm{v}$ and load voltage $12 \mathrm{v}$. A SDC SIMO converters are proposed for the designing and using for simple set of circuit blocks. A SDC SIMO converter is design for single input multiple output buck converter for light load efficiency. The proposed subsystem has 5 outputs each output has 10 watts total power is 50 watts the packaged converter is able to achieve the $73 \%$ peak efficiency with $.024 \mathrm{mV} / \mathrm{mA}$ cross regulation. Given more optimum packaging choices, simulation results show that efficiency of up to $86 \%$ and cross regulation of $0.04 \mathrm{mV} / \mathrm{mA}$ could be achieved with the proposed SDC control scheme. The energy in the inductor can be restored to the battery hence the proposed converter is more energy efficient.The load regulation is done without any complex control circuitry. The speed response of the controller is high due to state skipping. This can be used to power the Electronic loads such as cooling/heating system, lighting system, music system, etc., This can power different loads such as lamps, pumps with $12 \mathrm{~V}$ and provides supply to microcontroller with $5 \mathrm{~V}$.

\section{REFERENCES}

1. Tae Young Goh and Wai Tung Ng "Single Discharge Control for Single-Inductor Multiple-Output DC-DC Buck Converters" IEEE transactions on Power electronics volume:33, issue: 3, march 2018.

2. B. Arbetter, R. Erickson, and D. Maksimovic, "DC-DC Converter Design for Battery-Operated Systems," IEEE Power Electronics Specialist Conference, vol. 1, pp. 103- 109, 1995.

3. G. A. Rincon-Mora, and P. E. Allen, "A Low-Voltage, Low Quiescent Current, Low Drop-Out Regulator," IEEE Journal of Solid-State Circuits, vol. 33, pp. 36-44, 1998.

4. V. Gupta, G. A. Rincon-Mora, and P. Raha, "Analysis and Design of Monolithic, High PSR, Linear Regulators for SoC Applications," IEEE International SOC Conference, 2004, pp. 311-315.

5. D. Kwon, G. A. Rincon-Mora, "Single-Inductor-Multiple Output Switching DC- DC Converters," IEEE Transactions on Circuits and Systems-II: Express Briefs, vol. 56, no. 8, pp. 614-618, 2009.

6. M. Dongsheng, K. Wing-Hung, and T. Chi-Ying, "A pseudoCCM/DCM SIMO switching converter with freewheel switching," IEEE Journal of Solid-State Circuits, vol. 38, pp. 1007-1014, 2003.

7. T. L. Lee, J. K. O. Sin and P. C. H. Chan, "Scalability of QuasiHysteretic finite state machine control (FSM)-Based Digitally Controlled Single-Inductor Dual-String Buck LED Driver to Multiple Strings," in IEEE Transactions on Power Electronics, vol. 29, no. 1, pp. 501-513, Jan. 2014.

8. Miao Yang, Weifeng Sun, Shen Xu, Shengli Lu and Longxing Shi, "A $65 \mathrm{~nm} 10 \mathrm{MHz}$ single-inductor dualoutput switching buck converter with time-multiplexing control," 2011 9th IEEE International Conference on ASIC, Xiamen, 2011, pp. 870-873.

9. Daniel J. Fonseca, Daniel O. Navaresse, and Gary P. Moynihan. Simulation metamodeling through artificial neural networks. Engineering Applications of Artificial Intelligence, 16(3):177-183, 2003.

10. B. C. Cs'aji. Approximation with artificial neural networks. Faculty of Sciences, EtvsLornd University, Hungary, 24:48, 2001.

11. www.ti.com

\section{AUTHORS PROFILE}

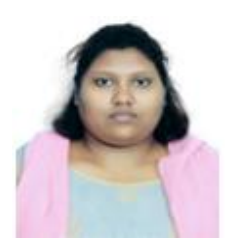

Pragnya R Mogha, PG Scholar, Department of Electrical and Electronics Engineering, PoojyaDoddappaAppa College of Engineering, Kalaburagi (Karnataka)-585102, India. E-mail: moghapragnya1931@gmail.com

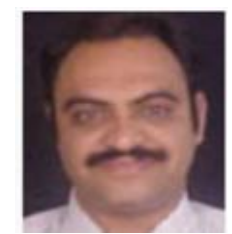

Manish Rathi, Associate Professor, Department of Electrical and Electronics Engineering, PoojyaDoddappaAppa College of Engineering, Kalaburagi (Karnataka)-585102, India. E-mail: manish.rathi277@gmail.com

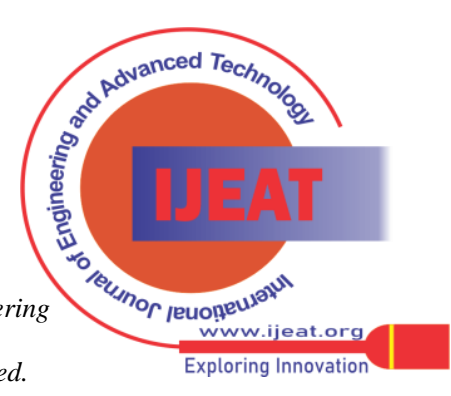

